\title{
Limited potential of resolvin D1 in treatment of cholestatic liver fibrosis
}

\author{
Kerstin Abshagen, Alexander Hartmann, Laura Grüner, Marie Liebig, Brigitte Vollmar \\ Institute for Experimental Surgery, University Medicine Rostock, Rostock, Germany \\ Contributions: (I) Conception and design: K Abshagen, B Vollmar; (II) Administrative support: K Abshagen, B Vollmar; (III) Provision of study \\ material or patients: None; (IV) Collection and assembly of data: A Hartmann, L Grüner, M Liebig; (V) Data analysis and interpretation: K \\ Abshagen, A Hartmann, L Grüner, M Liebig; (VI) Manuscript writing: All authors; (VII) Final approval of manuscript: All authors. \\ Correspondence to: Kerstin Abshagen, PhD. Institute for Experimental Surgery, University Medicine Rostock, Schillingallee 69a, 18057 Rostock, \\ Germany. Email: kerstin.abshagen@uni-rostock.de.
}

\begin{abstract}
Background: Several studies suggest a role for EPA- and DHA-derived pro-resolving mediators like resolvins in reversing metabolic and inflammatory disturbances seen in various chronic diseases. Here, we investigated the effects of resolvin D1 (RvD1) on bile duct ligation (BDL)-induced cholestatic liver injury.

Methods: Mice were treated daily with RvD1 or $0.1 \%$ ethanol (control) from the day of BDL until the final observation time points. Blood and liver tissue were collected 2, 5 and 14 days after BDL for different analyses.

Results: RvD1 treatment of mice had no impact on the extent of cholestatic liver injury upon BDL, neither in the acute phase nor in the progressive state of liver fibrosis. Although RvD1 treatment resulted in a significantly reduced activity of hepatic stellate cells as well as reduced deposition of extracellular matrix 2 days after BDL, mice were not protected from inflammation and further fibrosis progression.

Conclusions: These data indicate that RvD1 has a limited therapeutic potential to treat cholestatic liver diseases, as it has no significant impact on regression of hepatic necroinflammation and fibrotic changes in bile duct-ligated mice.
\end{abstract}

Keywords: Lipid mediator; n-3 polyunsaturated fatty acids; bile duct ligation (BDL); mouse model

Submitted Apr 05, 2019. Accepted for publication Jul 12, 2019.

doi: $10.21037 /$ hbsn.2019.08.07

View this article at: http://dx.doi.org/10.21037/hbsn.2019.08.07

\section{Introduction}

Worldwide incidence of chronic liver disease, including cholestatic diseases like primary biliary cirrhosis and primary sclerosing cholangitis, is rapidly increasing and because of limited therapeutic options it is a significant health problem (1). Its pathogenesis is associated with inflammation characterized by increased accumulation of macrophages and other inflammatory cells as well as activation of extracellular matrix-producing cells. However, regression of liver fibrosis is a known phenomenon, which has been observed after cure of the underlying disease (2).

Changes in dietary habits and nutrition might be an effective and simple strategy for the treatment and even for prevention of chronic liver diseases. Among protectins and maresins, resolvins $(\mathrm{Rv})$ belong to the family of specialized pro-resolving lipid mediators (SPMs), which are naturally occurring bioactive omega-3 polyunsaturated fatty acid (n-3 PUFA)-derived metabolites. Marine food containing n-3 PUFAs, namely eicosapentaenoic acid (EPA) and docosahexaenoic acid (DHA), and their metabolites have been shown to exert anti-inflammatory, cytoprotective and anti-steatotic effects in multiple preclinical models of disease (3-9), including steatosis, nonalcoholic steatohepatitis (NASH) and hepatic fibrosis (10-17). Of most interest, regular fish consumption and dietary intake of n-3 PUFAs were associated with a lower risk for hepatocellular carcinoma $(18,19)$. However, clinical trials using n-3 PUFA 
rich diets have produced discrepant findings (20).

DHA serves as a substrate for the formation of D-series resolvins (21), which are generated at sites of inflammation and act locally (22). DHA administration has been shown to result in significant higher levels of $\mathrm{RvD} 1$ and $\mathrm{RvD} 2$ in the hepatic tissue (23). Resolvins display potent proresolving and anti-inflammatory activities (24) and, thus, act to limit tissue damage $(5,25)$. In fact, treatment of mice with RvD1/D2 ameliorated ischemia/reperfusion-induced hepatocellular damage (26-28), prevented concanavalin A-induced liver injury and the changes of hepatitis to liver cancer in mice (29) and attenuated acetaminophen-induced hepatotoxicity (30). Mechanisms underlying these proresolving actions include altered cell membrane composition and membrane fluidity, inhibition of chemotaxis and transendothelial migration of neutrophils $(21,30)$, phenotypic changes of macrophages $(31,32)$, inhibition of NFאB (nuclear factor kappa B) activation $(7,29,33)$ and thus, reduced expression of inflammatory genes $(4,24,33)$.

Overall, the scientific evidence of RvD1 as an attractive therapeutic in liver diseases prompted us to study its potential to attenuate bile duct ligation (BDL)-induced liver injury in mice by suppressing inflammation and fibrogenesis.

We present the following article in accordance with the ARRIVE reporting checklist (available at http://dx.doi. org/10.21037/hbsn.2019.08.07).

\section{Methods}

\section{Mice}

Male Balb/c mice (Charles River Laboratories) at an age of 12 to 16 weeks and a body weight (bw) of approximately 25-30 g were kept at a $12 \mathrm{~h}$ day and night cycle on water and standard laboratory chow ad libitum. Experiments were approved by the local government Landesamt für Landwirtschaft, Lebensmittelsicherheit und Fischerei Mecklenburg-Vorpommern (LALLF M-V/TSD/7221.31.1-028/12) and conducted in accordance with the German legislation on protection of animals and EU-directive 2010/63/EU.

\section{Surgical procedure and experimental groups}

Mice underwent ligation of the common bile duct under isoflurane inhalation (1.5 vol\%) as previously described by our group (34-36). After recovery from anesthesia and surgery under a red warming lamp, mice were treated with metamizole $(0.8 \mathrm{~mL} / 500 \mathrm{~mL})$ as analgesic in their drinking water until the final observation time points. Mice were daily injected intraperitoneally with resolvin D1 (RvD1) ( $2 \mathrm{ng} / \mathrm{g}$ bw in $0.1 \%$ ethanol; RvD1). RvD1 was purchased from Cayman Chemical (BIOMOL GmbH, Hamburg, Germany, \#10012554). Starting directly after BDL until the indicated time points, control animals received equivalent volumes of $0.1 \%$ ethanol (control). Mice were sacrificed under ketamine/xylazine anesthesia $(90 / 7 \mathrm{mg} / \mathrm{kg}$ bw ip) at postoperative days 2, 5 and 14 (10 animals per time point and group) and blood as well as liver tissue samples were taken and processed for subsequent analyses. To analyze the regenerative response, 5-bromo-2-deoxyuridine (BrdU; $50 \mathrm{mg} / \mathrm{kg}$ bw ip) was injected $1 \mathrm{~h}$ prior to harvest of liver tissue. BrdU incorporation into the DNA was analyzed by immunohistochemistry.

\section{Hematological measurements and plasma enzyme levels}

Blood samples were collected at the final time points by retrobulbar sinus puncture. Plasma activities of alanine aminotransferase (ALT) and glutamate dehydrogenase (GLDH) as indicators of hepatocellular disintegration and necrosis as well as plasma concentration of total bilirubin (Bili-T) and alkaline phosphatase (ALP) as cholestasis parameters were measured spectrophotometrically using cobas c 111 analyzer (Roche Diagnostics; Rotkreuz, Switzerland) according to the manufacturer's instructions.

\section{Histopathology and image analysis}

Formalin fixed liver tissue samples were embedded in paraffin and sliced into $5 \mu \mathrm{m}$ thick sections. For routine examination and quantification of necrotic areas (bile infarcts) tissue slices were stained with hematoxylin and eosin (H\&E). Sirius red staining was used for quantification of collagen deposition. All samples from a series of experiments were stained simultaneously and evaluated in a blinded manner. For histomorphometric analyses, images of 20 random low power fields (100x magnification, Olympus BX 51, Hamburg, Germany) were taken with a Color View II FW camera (Color View, Munich, Germany) and evaluated using Adobe Photoshop CS5 12.0.4 (Adobe, San José, California, USA) or ImageJ 1.47v. Fibrosis deposition was quantified as percentage of Sirius red-stained area compared with the total section area. Bile infarcts were quantified in $\mathrm{H} \& \mathrm{E}$-stained sections in a similar manner and 
the percentage of the focal necrosis area to the total liver section area was assessed. For the assessment of granulocyte infiltration, paraffin-embedded liver tissue sections were stained for chloroacetate esterase (CAE) with naphthol AS-D chloroacetate (Sigma-Aldrich, St. Louis, Missouri, USA) and quantified as described by Liebig et al. (37).

\section{Immunobistochemistry}

Five $\mu \mathrm{m}$ thick paraffin embedded liver slices were immuno-stained for F4/80. The slides were incubated with the appropriate primary antibody $1 \alpha$-rabbit-antimouse-Collagen (1:200, Abcam 34710) overnight at $4{ }^{\circ} \mathrm{C}$. The secondary antibody (F4/80: goat-anti-rabbit-AP, 1:200, Santa Cruz SC2021) was incubated for $1 \mathrm{~h}$ at room temperature (RT). Signal detection was performed by using Permanent Red (Dako) or 3,3'-diaminobenzidine (DAB, Dako). Furthermore, nuclei were counterstained with hemalaun. F4/80-positive cells were counted in a blinded manner within 30 consecutive high power fields (HPF) $(\times 40$ objective, numerical aperture 0.65 ) and given as cells/HPF.

For analyzing DNA-incorporated BrdU in liver cells, $5 \mu \mathrm{m}$ sections collected on poly-L-lysine-coated glass slides were incubated with monoclonal mouse anti-BrdU antibody (1:50; M0744, Dako) overnight at $4{ }^{\circ} \mathrm{C}$, followed by HRPconjugated goat anti-mouse immunoglobin (LSAB kit plus; Dako). Sites of peroxidase-binding were detected by DAB (Dako). Sections were counterstained with hemalaun. BrdUpositive hepatocellular nuclei and non-parenchymal cells were counted in a blinded manner within 30 consecutive high power fields $(\mathrm{HPF})(\times 40$ objective, numerical aperture $0.65)$ and given as cells/HPF.

\section{Western blot analysis and quantification}

Protein expression was analyzed as described by our group (37) using $40 \mu \mathrm{g}$ extracted protein per lane and the following primary antibodies: anti- $\alpha$ SMA (alpha smooth muscle actin) (A2547, clone 1A4; 1:1,000; Sigma Aldrich Chemical Company, Steinheim, Germany) and antiGAPDH (glycerinaldehyd-3-phosphat-dehydrogenase) (1:20,000; MAB374, Merck Millipore Chemicals GmbH, Schwalbach, Germany). The corresponding HRPconjugated secondary anti-rabbit (1:10,000; Cell Signaling) and anti-mouse (1:40,000; Sigma) antibodies were incubated for $1 \mathrm{~h}$ at RT and were detected using ECL Western blot reagents (Amersham Biosciences). Quantitative analyses of aSMA expression were performed densitometrically using the Image Lab software 6.0 (Bio-Rad Laboratories GmbH, Munich, Germany). All signals were normalized to the GAPDH signals of the corresponding sample.

\section{qRT-PCR analysis}

Total RNA was isolated from snap frozen liver tissue lysates using RNeasy Mini Kit including on column genomic DNA digestion with RNase free DNase Set (Qiagen, Hilden, Germany). For qRT-PCR $2 \mu \mathrm{g}$ RNA was reverse transcribed to cDNA using Oligo(dT)18 primer (New England Biolabs GmbH) and Superscript II RNaseH-Reverse Transcriptase (Invitrogen, Life Technologies, Darmstadt, Germany). Real-time PCR gene expression analysis was performed by the iTaq ${ }^{\mathrm{TM}}$ Universal SYBR ${ }^{\circledR}$ Green Supermix (Bio-Rad Laboratories, Hercules, California, USA) applying the Bio-Rad iQ5 system (Bio-Rad Laboratories, Hercules, California, USA) and the following primers for Collagen 1 $\alpha$ : for 5'-GAAACCCGAGGTATGCTTGA-3', rev 5'-GACCAGGAGGACCAGGAAGT-3'; Tnf $\alpha$ : for 5'-AGGCTCTGGAGAACAGCACAT-3', rev 5'-TGGCTTCTCTTCCTGCACCAAA-3'; Gapdb: for 5'-GAATTTGCCGTGAGTGGAGT -3', rev 5'-CGTCCCGTAGACAAAATGGT-3'. All data were calculated by using the comparative $2^{-\Delta \Delta \mathrm{Ct}}$ method and expression values were normalized to the expression levels of the housekeeping gene Gapdh. Target gene expression was compared to a liver tissue pool obtained from healthy Balb/c mice.

\section{Statistical analysis}

All statistical analyses were performed using GraphPad Prism 8 (GraphPad Software, La Jolla, California, USA). In normally distributed data sets outliers were identified using Grubb's test and were excluded if significance was $\mathrm{P}<0.05$. The group difference within each time point was assessed by $t$-test and is indicated in the graphs as follows: ${ }^{*} \mathrm{P}<0.05$, ${ }^{* *} \mathrm{P}<0.01,{ }^{* * *} \mathrm{P}<0.001$. Statistical significance was set at $\mathrm{P}<0.05$. All data are presented as box plots indicating the median, the interquartile range in form of a box, and the minimum and maximum as whiskers.

\section{Results}

\section{Cholestatic liver injury}

First, we analyzed the effect of RvD1 on the BDL-induced 

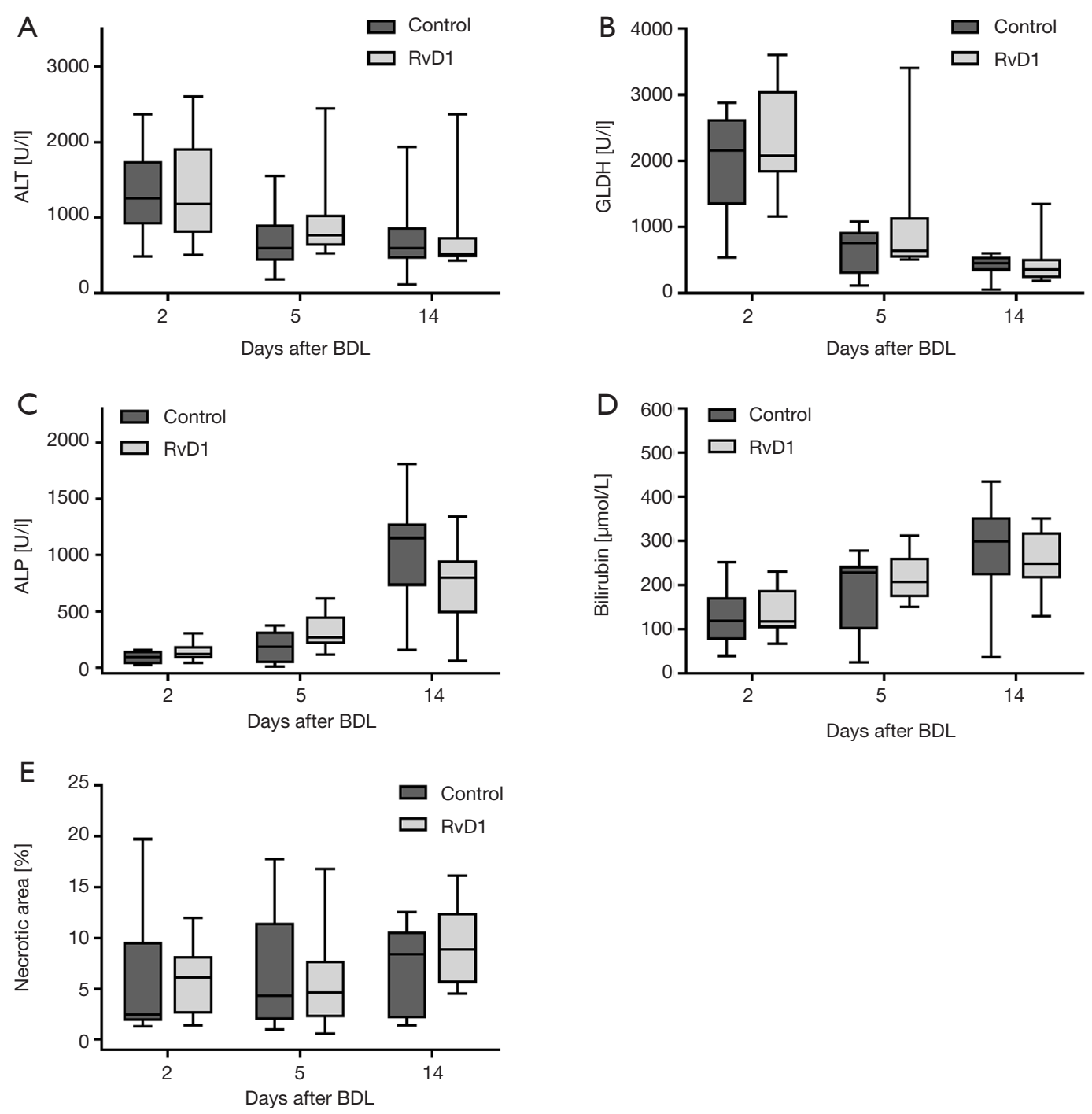

Figure 1 Analysis of plasma activities of (A) alanine aminotransferase (ALT), (B) glutamate dehydrogenase (GLDH) and (C) alkaline phosphatase (ALP) as well as (D) total plasma bilirubin level of resolvin D1 (RvD1) and ethanol (control)-treated mice 2, 5 and 14 days after BDL. (E) Quantification of bile infarct/necrotic area in H\&E-stained liver sections at multiple time points after BDL. Values are presented as box plots indicating the median, the interquartile range in form of a box, and the minimum and maximum as whiskers. Group differences were tested by $t$-test within each time point, $\mathrm{n}=9-10$ per time point and group. BDL, bile duct ligation.

liver injury (Figure 1). In both groups, activities of ALT and GLDH in plasma were pathologically high already at day 2 after BDL and declined thereafter in a similar manner (Figure 1A,B). The cholestasis parameters ALP and total bilirubin showed a constant rise above physiological values over the observation period of 14 days after BDL in controls as well as RvD1-treated mice (Figure 1C,D). Accordingly, we observed no difference in necrotic area (bile infarcts) of H\&E-stained liver tissue slides between
RvD1-treated and control mice after BDL (Figure 1E). In summary, treatment of mice with RvD1 had no impact on the extent of cholestatic liver injury upon BDL, neither in the acute phase after BDL nor in the progressive state of liver fibrosis.

\section{Inflammatory response}

BDL-induced injury was associated by a marked increase 
A

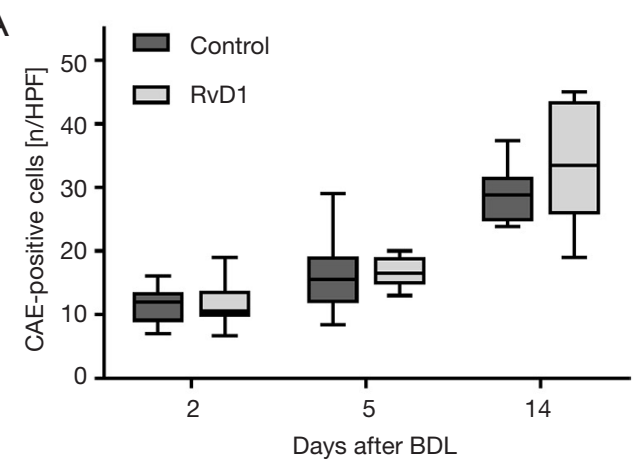

C

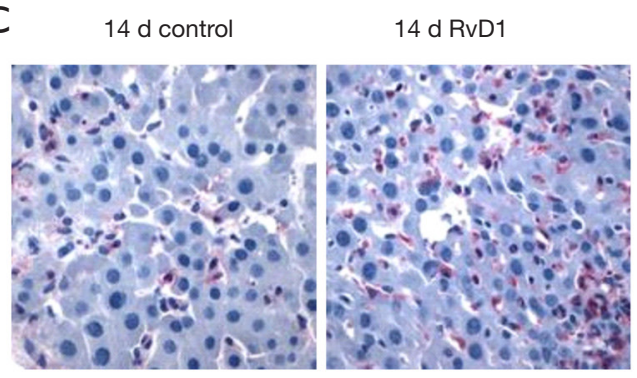

B
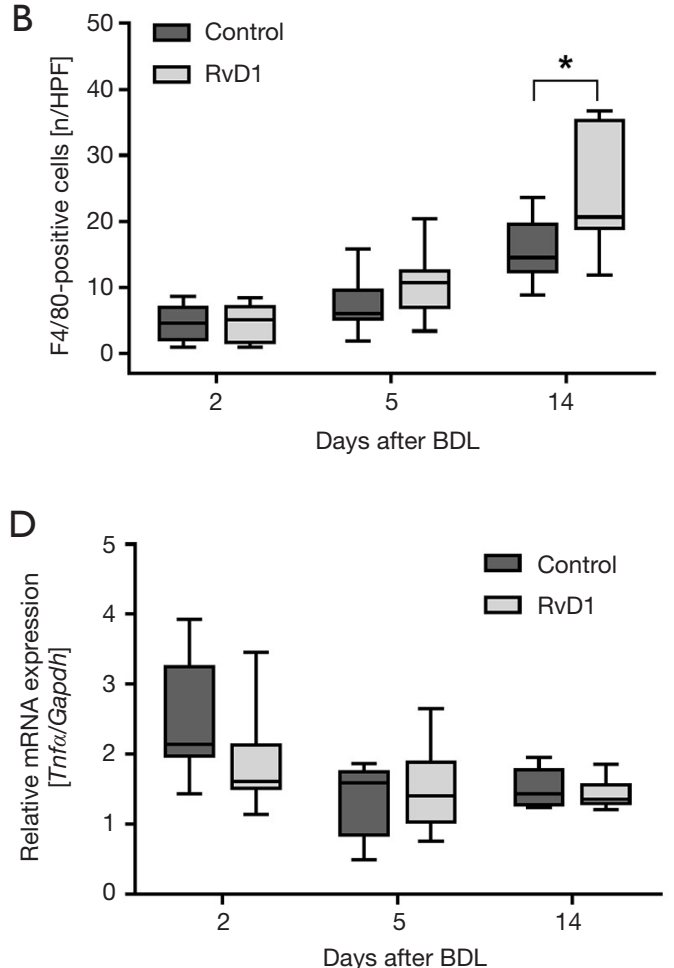

Figure 2 Quantitative analysis of (A) CAE and (B) F4/80-stained liver sections and (D) quantitative qRT-PCR analysis of Tnfa mRNA expression in livers of resolvin D1 (RvD1) and ethanol (control)-treated mice 2, 5 and 14 days after BDL. (C) Representative photomicrographs of F4/80 stained liver sections of control and RvD1 group $14 \mathrm{~d}$ after BDL (400× magnification). Values are presented as box plots indicating the median, the interquartile range in form of a box, and the minimum and maximum as whiskers. Group differences were tested by $t$-test within each time point. *, $\mathrm{P}<0.05$, n=9-10 per time point and group. BDL, bile duct ligation.

of inflammatory cells in liver tissue, like granulocytes (Figure 2A) and macrophages (Figure 2B,C) in both control and RvD1-treated mice. Interestingly, compared to controls a significantly higher number of $\mathrm{F} 4 / 80$-positive cells was observed in RvD1-treated mice 14 days after BDL (Figure 2B), whereas the number of CAE-positive cells did not differ significantly between both groups (Figure $2 A$ ). Additional analysis of mRNA expression of Tnfa (Figure 2D) revealed no significant differences of this marker for M1-polarized macrophages between the groups at any time point (Figure 2D). However, RvD1-treated mice showed a slightly reduced hepatic Tnf $\alpha$ mRNA expression at day 2 after BDL (Figure 2D).

\section{Activation of hepatic stellate cells (HSC) and fibrosis}

To study the extent of fibrosis, we examined the activation of HSC and quantified the amount of extracellular matrix in the liver (Figure 3). Of most interest, protein expression of aSMA, a marker of activated HSC, was significantly reduced in RvD1-treated mice at day 2 post BDL when compared to control (Figure $3 A, B$ ). Additionally, the aSMA protein expression trended to be reduced in RvD1-treated mice 14 days after BDL (Figure 3A). In consequence, 2 days after BDL livers of RvD1-treated mice showed significantly less deposition of extracellular matrix than control mice as shown by Sirius red histology (Figure 3C). However, this result could not be verified by mRNA expression analysis of Collagen $1 \alpha$, where no differences between the groups were observed at any time point (Figure 3D).

\section{Proliferative and regenerative response}

As seen by the growing number of proliferating cells over time, progression of fibrosis also induced a proliferative response of liver cells (Figure 4A,B). Both groups displayed almost equal numbers of BrdU-positive hepatocytes and non-parenchymal cells at all observation time points 
A

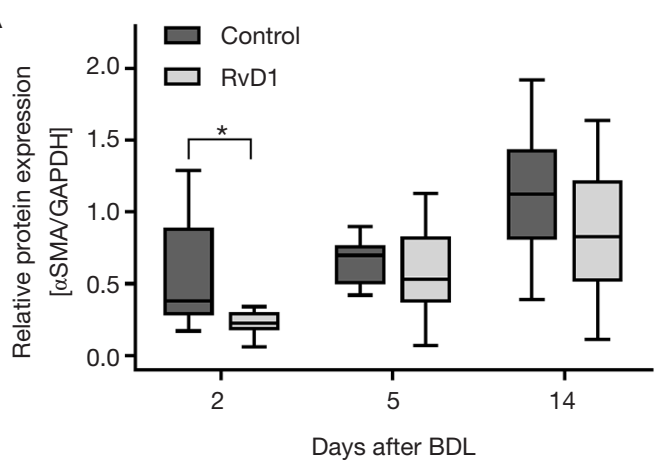

C

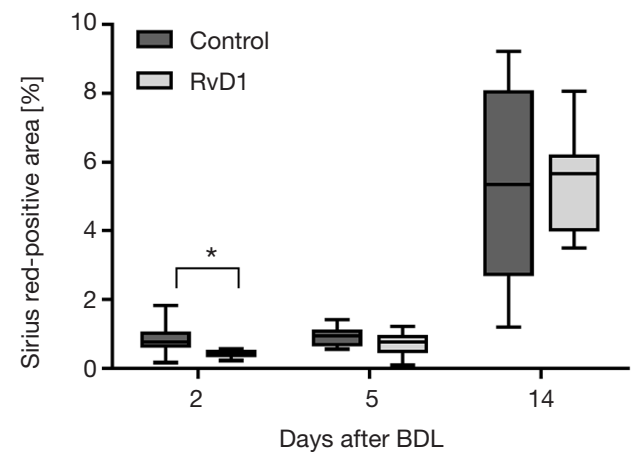

B
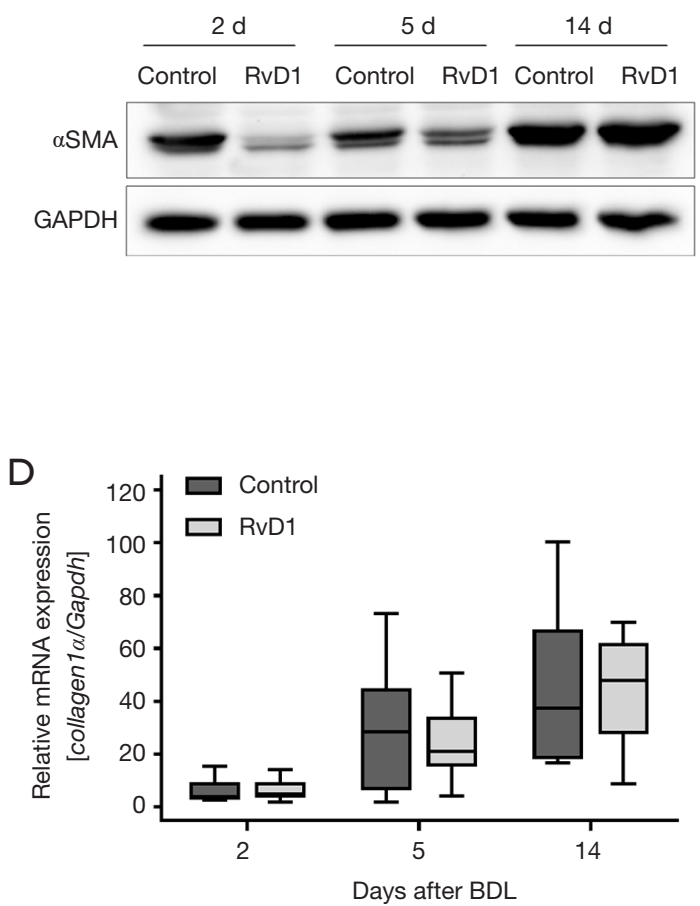

Figure 3 (A) Quantitative analysis of hepatic protein expression of $\alpha \mathrm{SMA}$ and (B) representative western blot images of $\alpha \mathrm{SMA}(42 \mathrm{kDa})$ and GAPDH (38 kDa) in livers of resolvin D1 (RvD1) and ethanol (control)-treated mice 2, 5 and 14 days after BDL. Quantitative analysis of (C) Sirius red-positive area in appropriately stained liver sections and (D) mRNA expression of collagen1 $\alpha$ of resolvin D1 (RvD1) and ethanol (control) treated mice 2, 5 and 14 days after BDL. Values are presented as box plots indicating the median, the interquartile range in form of a box, and the minimum and maximum as whiskers. Group differences were tested by $t$-test within each time point. *, $\mathrm{P}<0.05, \mathrm{n}=9-10$ per time point and group. BDL, bile duct ligation.

after BDL (Figure $4 A, B$ ). Similarly, the liver weight to body weight ratio, which gradually increased during fibrogenesis, was not affected by the treatment with RvD1 (Figure 4C).

\section{Discussion}

Conflicting results in terms of the efficiency of n-3 PUFA supplementation in prevention and therapy of chronic diseases exist. While animal experiments suggest that $\mathrm{n}-3$ PUFAs and their mediators may be promising therapeutic agents for liver disease, clinical trials addressing the effects of n-3 PUFAs are inconsistent, failing to show a clear evidence of efficacy (20). The obtained results varied because of different dietary composition, dosages of $n-3$ PUFAs and their mediators, n-3/n-6 PUFA ratios, duration, patient specific factors, exercise, compliance, sensitivity of methods and other factors. However, trials have indicated that n-3 PUFAs might be more beneficial in decreasing lipid load, than in decreasing other features of steatohepatitis or liver fibrosis $(20,38)$. As dietary supplementation with fish oil, containing high amounts of DHA and EPA, had beneficial effects in treatment of various inflammatory diseases and cancer $(19,22,29)$, n-3 PUFA-derived lipid mediators are also presumed to be important in the control of fibrogenesis. Furthermore, several studies have shown, that fat-1-transgenic mice, which are able to convert n- 6 to n-3 PUFAs and thus exhibit higher tissue levels of EPA and DHA as well as of resolvins and protectins, are protected from many chronic diseases, including inflammatory and neurological disorders, cancer, obesity and diabetes, osteoporosis as well as HFD-induced NAFLD $(9,13,39-42)$. Importantly, the anti-fibrotic potential of SPMs has been demonstrated in diverse preclinical models of hepatic, renal and pulmonary fibrosis $(6,10,16,29,43)$. In contrast to the well-established anti-inflammatory and pro-resolution 

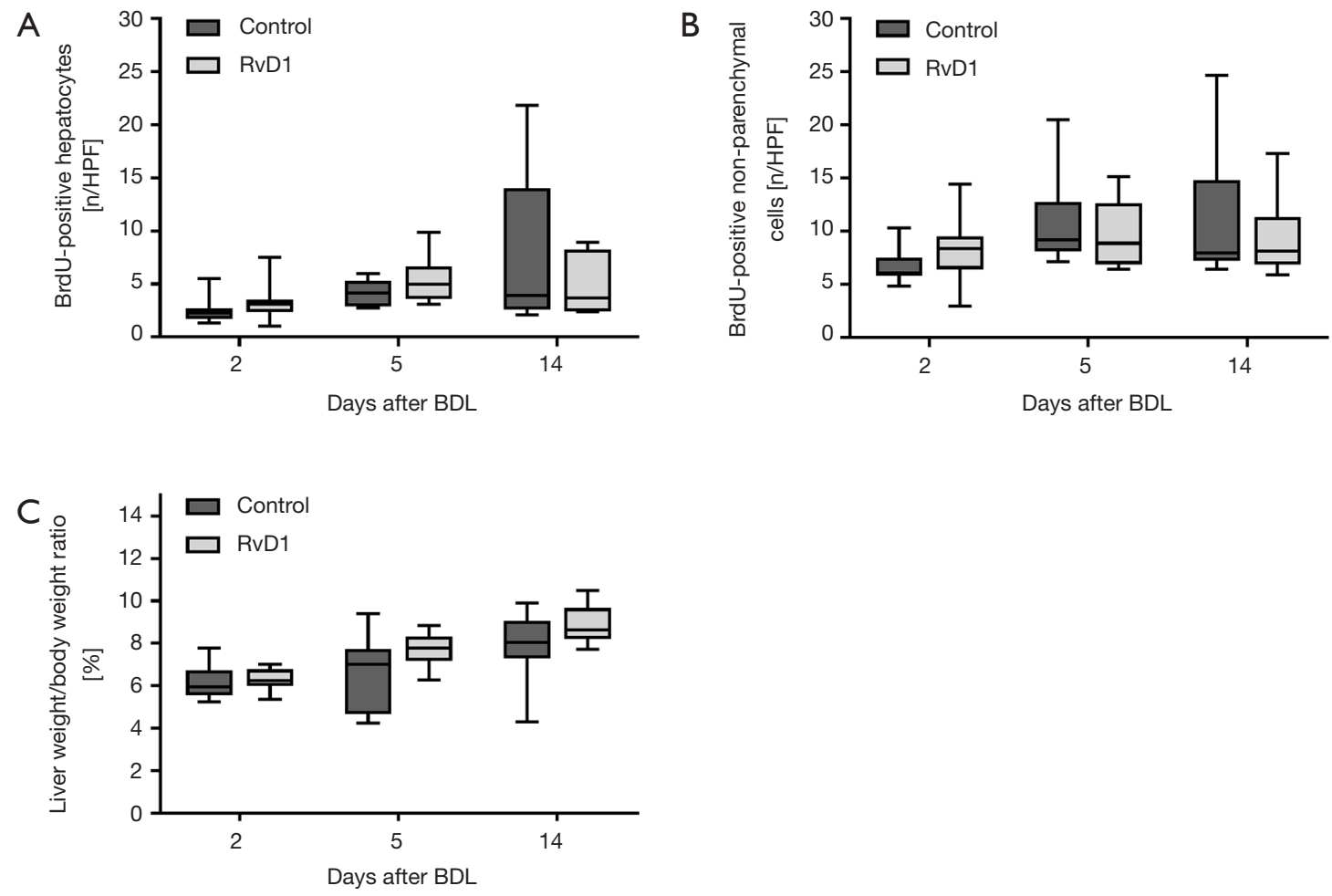

Figure 4 Number of (A) BrdU-positive hepatocytes and (B) non-parenchymal cells in immunohistochemically stained liver sections as well as (C) liver/body weight index of resolvin D1 (RvD1) and ethanol (control)-treated mice 2, 5 and 14 days after BDL. Values are presented as box plots indicating the median, the interquartile range in form of a box, and the minimum and maximum as whiskers. Group differences were tested by $t$-test within each time point. $n=9-10$ per time point and group. BDL, bile duct ligation.

properties of RvD1 in previous investigations $(4,28,44)$, administration of RvD1 in the current study had no significant beneficial effects on the disease process upon BDL except a transient attenuated fibrogenesis in the acute inflammatory phase.

It is known from rodent models as well as human biopsies that resolution of advanced liver fibrosis occurs after therapy, if the underlying fibrogenic stimuli are corrected and adequate pro-resolving cellular programs are activated (2). Furthermore, fibrosis regression is associated with a change in the number or activation status of extracellular matrixproducing cells (2). Previously, it has been shown that fish oil attenuates $\mathrm{CCl}_{4}$-induced liver fibrosis in mice by downregulation of the expression of pro-fibrogenic genes in HSC (17). Accordingly, we observed an attenuated HSC activation in the early phase of fibrogenesis in RvD1-treated mice, which resulted in transiently decreased extracellular matrix deposition. However, this positive effect of RvD1 disappeared with disease progression and increasing severity of disease.
Hepatic macrophages may also contribute to fibrosis resolution through a phenotypic switch (2). Recent studies suggest inflammatory macrophages as the target for the $n-3$ PUFA DHA as well as RvD1 $(32,45)$, thereby influencing the kinetics of hepatic macrophage accumulation. Thus, RvD1 treatment has been shown to result in enhanced switching from a pro-inflammatory, M1- to an anti-inflammatory, M2like phenotype $(26,31,32)$, finally stimulating macrophage uptake of apoptotic neutrophils (25). Due to the significantly increased number of F4/80-positive cells 14 days after BDL and simultaneously no augmented Tnf $\alpha$ mRNA expression, we suggest an induction of restorative M2-like macrophages rather than an intensified inflammation.

It is important to note that in our study, neutrophil infiltration (as assessed by CAE-staining) was not positively modulated by RvD1 treatment, which might be explained by limited RvD1 availability in liver tissue. Similarly, using a dose of $1.2 \mathrm{ng} / \mathrm{g}$ body weight RvE1 every day did not show any beneficial effects in NASH with neither steatosis grade nor expression of inflammatory or fibrotic genes being 
improved (46). As SPMs are known to exert their biological actions in the picomolar to nanomolar range $(10,16,45)$, a RvD1 dosage of $2 \mathrm{ng} / \mathrm{g}$ body weight (approximately $60 \mathrm{ng} /$ mouse) was used in the current study (47). It must be noted that most of the studies observing protective effects of resolvins used supraphysiological doses of RvD1/D2 or even combined different mediators $(7,11,30,32,48,49)$. For example, in a murine hind-limb ischemia/reperfusion model only at high doses of $1 \mu \mathrm{g}$ /mouse RvD1 tissue protection was seen by inhibited neutrophil infiltration (48). However, it is still unclear whether such high dosages would be tolerated clinically. Nevertheless, a limitation of our study is that we did not test various doses of RvD1. It also has to be considered that resolvins undergo local metabolic inactivation in vivo (21). Thus, metabolically stable and longer lasting resolvin mimetics/analogs prevent rapid inactivation and have been shown to display potent protective actions even at lower concentrations $(21,44)$. Additionally, the effect of resolvins depends on receptor usage and target cells. For example, it was shown that expression of the RvE1 receptor chemokine-like receptor 1 (CMKLR1) was decreased in murine fibrotic liver (16) and human fatty liver (50). The biological actions of RvD1 are mediated by specific binding to the receptor FPR2 (ALX) (45). Of interest, we detected protein expression of FPR2 in liver tissue homogenates, but without any significant changes upon BDL (data not shown).

As the benefits of n-3 PUFAs on diverse pathological conditions are very different and depend primarily on the n-3 PUFA content and n-6/n-3 PUFA ratio (9), it can also be suggested that the absolute level of RvD1 and its availability in liver tissue appear to be critical for managing inflammation and fibrogenesis during cholestatic liver injury. Further work and strategies are needed to comprehensively define the optimal pharmacological concentration of $\mathrm{RvD} 1$ to ensure availability of $\mathrm{RvD} 1$, particularly in liver macrophages and HSC, and to prolong the biological activity of RvD1.

\section{Acknowledgments}

We thank Maren Nerowski, Eva Lorbeer, Dorothea Frenz, Berit Blendow for their excellent technical assistance.

Funding: This work was supported by the Deutsche Forschungsgemeinschaft, Bonn-Bad Godesberg, Germany (AB 453/2-1).

\section{Footnote}

Reporting Checklist: The authors have completed the ARRIVE reporting checklist. Available at http://dx.doi. org/10.21037/hbsn.2019.08.07

Data Sharing Statement: Available at http://dx.doi. org/10.21037/hbsn.2019.08.07

Conflicts of Interest: All authors have completed the ICMJE uniform disclosure form (available at http://dx.doi. org/10.21037/hbsn.2019.08.07). The authors have no conflicts of interest to declare.

Ethical Statement: Experiments were approved by the local government Landesamt für Landwirtschaft, Lebensmittelsicherheit und Fischerei MecklenburgVorpommern (LALLF M-V/TSD/7221.3-1.1-028/12 and conducted in accordance with the German legislation on protection of animals and EU-directive 2010/63/EU). The authors are accountable for all aspects of the work in ensuring that questions related to the accuracy or integrity of any part of the work are appropriately investigated and resolved.

Open Access Statement: This is an Open Access article distributed in accordance with the Creative Commons Attribution-NonCommercial-NoDerivs 4.0 International License (CC BY-NC-ND 4.0), which permits the noncommercial replication and distribution of the article with the strict proviso that no changes or edits are made and the original work is properly cited (including links to both the formal publication through the relevant DOI and the license). See: https://creativecommons.org/licenses/by-nc-nd/4.0/.

\section{References}

1. Sclair SN, Little E, Levy C. Current Concepts in Primary Biliary Cirrhosis and Primary Sclerosing Cholangitis. Clin Transl Gastroenterol 2015;6:e109.

2. Ramachandran P, Iredale JP, Fallowfield JA. Resolution of Liver Fibrosis: Basic Mechanisms and Clinical Relevance. Semin Liver Dis 2015;35:119-31.

3. Nagatake T, Kunisawa J. Emerging roles of metabolites of $\omega 3$ and $\omega 6$ essential fatty acids in the control of intestinal inflammation. Int Immunol 2019;31:569-77. 
4. Serhan CN, Chiang N, Van Dyke TE. Resolving inflammation: dual lipid mediators. Nat Rev Immunol 2008;8:349-61.

5. Calder PC. Marine omega- 3 fatty acids and in $\mathrm{flammatory}$ processes: Effects, mechanisms and clinical relevance. Biochim Biophys Acta 2015;1851:469-84.

6. Liu Y, Zhou D, Long FW, et al. Resolvin D1 protects against inflammation in experimental acute pancreatitis and associated lung injury. Am J Physiol Gastrointest Liver Physiol 2016;310:G303-9.

7. Liao Z, Dong J, Wu W, et al. Resolvin D1 attenuates inflammation in lipopolysaccharide-induced acute lung injury through a process involving the PPAR $\gamma / \mathrm{NF}-\kappa \mathrm{B}$ pathway. Respir Res 2012;13:110.

8. Chen J, Shetty S, Zhang P, et al. Aspirin-triggered resolvin D1 down-regulates inflammatory responses and protects against endotoxin-induced acute kidney injury. Toxicol Appl Pharmacol 2014;277:118-23.

9. Li J, Li FR, Wei D, et al. Endogenous Omega-3 Polyunsaturated Fatty Acid Production Confers Resistance to Obesity, Dyslipidemia, and Diabetes in Mice. Mol Endocrinol 2014;28:1316-28.

10. Musso G, Gambino R, Cassader M, et al. Specialized Proresolving Mediators: Enhancing Nonalcoholic Steatohepatitis and Fibrosis Resolution. Trends Pharmacol Sci 2018;39:387-401.

11. Echeverría F, Valenzuela R, Espinosa A, et al. Reduction of high-fat diet-induced liver proinflammatory state by eicosapentaenoic acid plus hydroxytyrosol supplementation: involvement of resolvins $\mathrm{RvE} 1 / 2$ and RvD1/2. J Nutr Biochem 2019;63:35-43.

12. de Castro GS, Calder PC. Non-alcoholic fatty liver disease and its treatment with n-3 polyunsaturated fatty acids. Clin Nutr 2018;37:37-55.

13. Kim EH, Bae JS, Baik KB, et al. Endogenously synthesized n-3 polyunsaturated fatty acids in fat- 1 mice ameliorate high-fat diet-induced non-alcoholic fatty liver disease. Biochem Pharmacol 2012;84:1359-65.

14. Kajikawa S, Harada T, Kawashima A, et al. Highly Purified Eicosapentaenoic Acid Ethyl Ester Prevents Development of Steatosis and Hepatic Fibrosis in Rats. Dig Dis Sci 2010;55:631-41.

15. González-Périz A, Horrillo R, Gronert K, et al. Obesityinduced insulin resistance and hepatic steatosis are alleviated by omega- 3 fatty acids: a role for resolvins and protectins. FASEB J 2009;23:1946-57.

16. Qiu W, Guo K, Yi L, et al. Resolvin E1 reduces hepatic fibrosis in mice with Schistosoma japonicum infection. Exp
Ther Med 2014;7:1481-5.

17. Zhang K, Chang Y, Shi Z, et al. $\omega-3$ PUFAs ameliorate liver fibrosis and inhibit hepatic stellate cells proliferation and activation by promoting YAP/TAZ degradation. Sci Rep 2016;6:30029.

18. Gao M, Sun K, Guo M, et al. Fish consumption and n-3 polyunsaturated fatty acids , and risk of hepatocellular carcinoma: systematic review and meta-analysis. Cancer Causes Control 2015;26:367-76.

19. Jump DB, Depner CM, Tripathy S, et al. Potential for Dietary $\omega-3$ Fatty Acids to Prevent Nonalcoholic Fatty Liver Disease and Reduce the Risk of Primary Liver Cancer. Adv Nutr 2015;6:694-702.

20. Scorletti E, Byrne CD. Omega-3 fatty acids and nonalcoholic fatty liver disease: Evidence of e fficacy and mechanism of action. Mol Aspects Med 2018;64:135-46.

21. Sun YP, Oh SF, Uddin J, et al. Resolvin D1 and Its Aspirintriggered 17 R Epimer. J Biol Chem 2007;282:9323-34.

22. Serhan CN, Petasis NA. Resolvins and Protectins in Inflammation-Resolution. Chem Rev 2011;111:5922-43.

23. Videla LA, Vargas R, Valenzuela R, et al. Combined administration of docosahexaenoic acid and thyroid hormone synergistically enhances rat liver levels of resolvins RvD1 and RvD2. Prostaglandins Leukot Essent Fatty Acids 2019;140:42-6.

24. Serhan CN. Novel Pro-Resolving Lipid Mediators in Inflammation Are Leads for Resolution Physiology. Nature 2014;510:92-101.

25. Serhan CN, Chiang N, Van Dyke T. Resolving inflammation: dual anti-inflammatory and pro-resolution lipid mediators. Nat Rev Immunol 2008;8:349-61.

26. Kang JW, Lee SM. Resolvin D1 protects the liver from ischemia/reperfusion injury by enhancing: M2 macrophage polarization and efferocytosis. Biochim Biophys Acta 2016;1861:1025-35.

27. Zhang T, Xiu HH, Liu JX, et al. Protective effect of aspirin-triggered resolvin D1 on hepatic ischemia/ reperfusion injury in rats: The role of miR-146b. Int Immunopharmacol 2017;51:140-7.

28. Kang JW, Choi HS, Lee SM. Resolvin D1 attenuates liver ischaemia/reperfusion injury through modulating thioredoxin 2-mediated mitochondrial quality control. Br J Pharmacol 2018;175:2441-53.

29. Kuang H, Hua X, Zhou J, et al. Resolvin D1 and E1 alleviate the progress of hepatitis toward liver cancer in long-term concanavalin A-induced mice through inhibition of NF-אB activity. Oncol Rep 2016;35:307-17.

30. Patel SJ, Luther J, Bohr S, et al. A Novel Resolvin-Based 
Strategy for Limiting Acetaminophen Hepatotoxicity. Clin Transl Gastroenterol 2016;(7):e153.

31. Titos E, Rius B, González-Périz A, et al. Resolvin D1 and Its Precursor Docosahexaenoic Acid Promote Resolution of Adipose Tissue Inflammation by Eliciting Macrophage Polarization toward an M2-Like Phenotype. J Immunol 2011;187:5408-18.

32. Rius B, Titos E, Morán-Salvador E, et al. Resolvin D1 primes the resolution process initiated by calorie restriction in obesity-induced steatohepatitis. FASEB J 2014;28:836-48.

33. Lee HN, Kundu JK, Cha YN, et al. Resolvin D1 stimulates efferocytosis through p50/p50-mediated suppression of tumor necrosis factor- $\alpha$ expression. J Cell Sci 2013;126:4037-47.

34. Abshagen K, König M, Hoppe A, et al. Pathobiochemical signatures of cholestatic liver disease in bile duct ligated mice. BMC Syst Biol 2015;9:83.

35. Abshagen K, Brensel M, Genz B, et al. Foxf1 siRNA delivery to hepatic stellate cells by DBTC lipoplex formulations ameliorates fibrosis in livers of bile duct ligated mice. Curr Gene Ther 2015;15:215-27.

36. Abshagen K, Senne M, Genz B, et al. Differential Effects of Axin2 Deficiency on the Fibrogenic and Regenerative Response in Livers of Bile Duct-Ligated Mice. Eur Surg Res 2015;55:328-40.

37. Liebig M, Hassanzada A, Kämmerling M, et al. Microcirculatory disturbances and cellular changes during progression of hepatic steatosis to liver tumors. Exp Biol Med (Maywood) 2018;243:1-12.

38. Kelley NS. Treatment of Nonalcoholic Fatty Liver Disease with Long-Chain n-3 Polyunsaturated Fatty Acids in Humans. Metab Syndr Relat Disord 2016;14:417-30.

39. Kang JX. The Omega-6/Omega-3 Fatty Acid Ratio in Chronic Diseases: Animal Models and Molecular Aspects. World Rev Nutr Diet 2011;102:22-9.

40. Weylandt KH, Krause LF, Gomolka B, et al. Suppressed liver tumorigenesis in fat-1 mice with elevated omega-3 fatty acids is associated with increased omega-3 derived lipid mediators and reduced TNF- $\alpha$. Carcinogenesis 2011;32:897-903.

41. Pai VJ, Wang B, Li X, et al. Transgenic Mice Convert Carbohydrates to Essential Fatty Acids. PLoS One 2014;9:e97637.

42. Heiker JT, Klöting N. Replication Initiator 1 in Adipose Tissue Function and Human Obesity. In: Vitamins and Hormones 2013:97-105.

43. Eickmeier O, Fussbroich D, Mueller K, et al. Pro-resolving lipid mediator Resolvin D1 serves as a marker of lung disease in cystic fibrosis. PLoS One 2017;12:e0171249.

44. Serhan CN, Krishnamoorthy S, Recchiuti A, et al. Novel Anti-Inflammatory -- Pro-Resolving Mediators and Their Receptors. Curr Top Med Chem 2011;11:629-47.

45. Krishnamoorthy S, Recchiuti A, Chiang N, et al. Resolvin D1 binds human phagocytes with evidence for proresolving receptors. PNAS 2010;107:1660-5.

46. Pohl R, Rein-Fischboeck L, Meier EM, et al. Resolvin E1 and chemerin C15 peptide do not improve rodent nonalcoholic steatohepatitis. Exp Mol Pathol 2015;98:295-9.

47. Hellmann J, Tang Y, Kosuri M, et al. Resolvin D1 decreases adipose tissue macrophage accumulation and improves insulin sensitivity in obese-diabetic mice. FASEB J 2011;25:2399-407.

48. Kasuga K, Yang R, Porter T, et al. Rapid Appearance of Resolvin Precursors in Inflammatory Exudates: Novel Mechanisms in Resolution. J Immunol 2008;181:8677-87.

49. Liu Y, Zhou D, Long F, et al. Resolvin D1 protects against inflammation in experimental acute pancreatitis and associated lung injury. Am J Physiol Gastrointest Liver Physiol 2016;310:G303-9.

50. Wanninger J, Bauer S, Eisinger K, et al. Adiponectin upregulates hepatocyte CMKLR1 which is reduced in human fatty liver. Mol Cell Endocrinol 2012;349:248-54.

Cite this article as: Abshagen K, Hartmann A, Grüner L, Liebig M, Vollmar B. Limited potential of resolvin D1 in treatment of cholestatic liver fibrosis. HepatoBiliary Surg Nutr 2020;9(5):587-596. doi: 10.21037/hbsn.2019.08.07 